Chapter III

Review Papers

Globular Clusters in Nearby Galaxies 


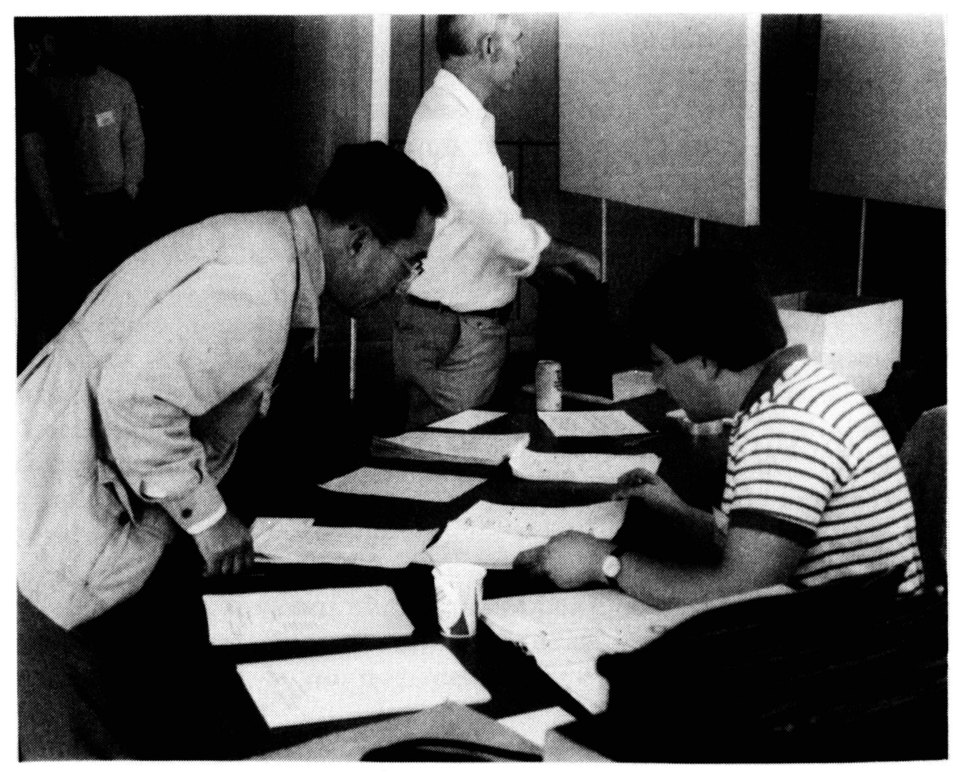

Bruce Carney studies a particulary detailed poster after registering

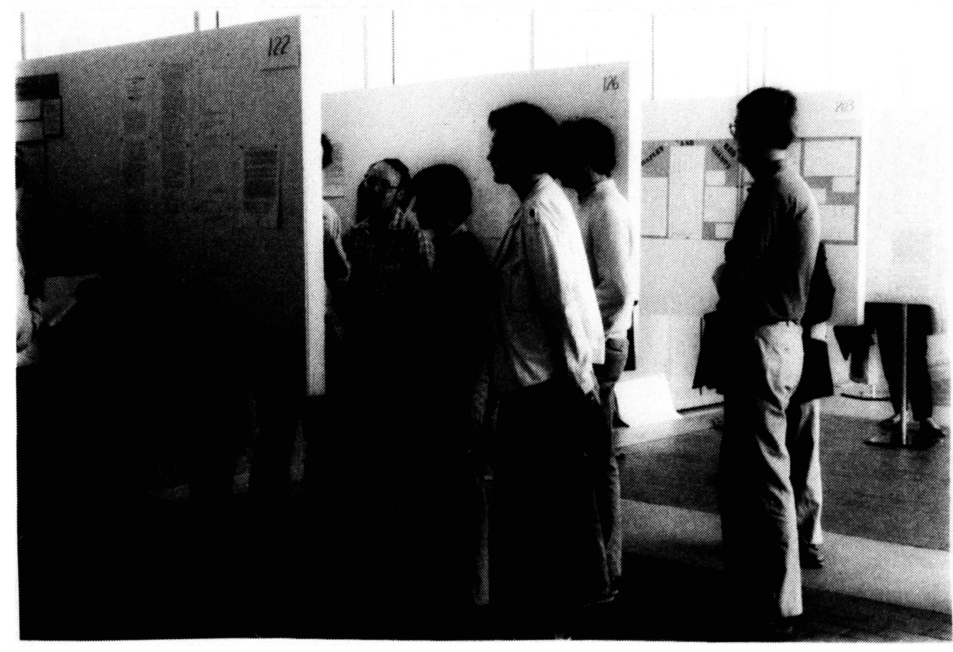

Gary DaCosta and colleagues studying posters 


\title{
OLD GLOBULAR CLUSTERS IN THE MAGELLANIC CLOUDS
}

\author{
J. A. Graham \\ Dept. of Terrestrial Magnetism \\ Carnegie Institution of Washington
}

\begin{abstract}
The old globular clusters in the Magellanic Clouds are important links between our understanding of globular clusters in our own galaxy and similar unresolved objects in more distant galaxies. The Cloud clusters spread over a large range in age. Several contain RR Lyrae variable stars. High welght abundance data are needed for individual cluster members as well as deeper color-magnitude diagrams.
\end{abstract}

\section{INTRODUCTION}

Harlow Shapley's contribution to Magellanic Cloud research was immense. Under his direction, the Harvard College Observatory dominated this field at a time when few other observatories realized its importance. Shapley's vision was clear and far-seeing. "In more ways than one," he wrote, "the Magellanic Clouds serve as a gateway to the Metagalaxy - to the outer and overall aggregate of galaxies" (Shapley 1956). In his book, Star Clusters (Shapley 1930), he reported how Miss Cannon had noted that many of the Cloud globular clusters had surprisingly early spectral types; a characteristic which will be much discussed at this meeting. Shapley's interest in the Magellanic Clouds and his drive towards their exploration continued through his years as Director at Harvard. However the road was not always easy and progress was limited by the instrumentation available at the time. For example, without photoelectric standards of brightness and color, it was difficult to do much towards constructing the color-magnitude diagrams of the quality we have come to take for granted.

In the context of this meeting, the Cloud clusters are indeed links in our understanding of the Galaxy and of the Universe as a whole. We can easily resolve the component stars in a Cloud cluster for individual studies. In a parallel way, we can examine the integrated brightness, spectrum and velocity of the cluster as a unit; something that can be quite hard to do for nearby objects. But for the clusters in more distant galaxies, this latter type of measurement is of ten all we can do and prior understanding of nearby examples is essential. 


\section{IDENTIFICATION OF OLD CLUSTERS}

There are not very many old globular clusters in the Magellanic Clouds in the sense that we understand the term in the Galaxy. One can easily count them of $f$ on the fingers of both hands. This makes my job today a fairly easy one but the next speaker, Ed Olszewski, has a much harder task. Some months ago, Ed and I decided that the borderline age should be $10 \mathrm{Gyr}$ with Ed concentrating on the intermediate age clusters which are of a type unknown in our galaxy and, as well, dominate the statistics. Because of the relative rarity of old clusters, it is important to review how they can be identified. In this group, every addition counts. We shall group the discovery process under 3 headings.

\subsection{Color-Magnitude Diagrams}

Historically, the successful assembly of color-magnitude diagrams for Magellanic Cloud clusters has been the spur to further investigation of their characteristics. The technique here was pioneered by Arp (1961) and by Gascoigne $(1962,1966)$. It was shown that while some clusters looked very similar to old globular clusters in our galaxy, others resembled more the intermediate age open clusters while a third class appeared to have no counterparts in the Galaxy at all. Until the arrival of fast linear detecting arrays like the CCD, this work was limited by the inability to push below the limits of the main-sequence turn-of $f$. Now that this can easily be done, the whole history of the cluster system in the Magellanic Clouds is coming into sharper focus. A good example is the cluster Hodge 11. Earlier work (Gascoigne 1966, Walker 1979) was not able to distinguish a blue horizontal branch from a blue main sequence. As we know now, the presence of a background component of field stars with different age contributed to the confusion. Now it is very clear and agreed upon by everybody that Hodge 11 is one of the oldest and most metal-poor of all globulars in the Clouds. (Andersen, Blecha and Walker 1984, Stryker, Nemec, Hesser and McClure 1984). The most recent color-magnitude diagrams for old clusters support the "short" distance scale for the Magellanic Clouds (Andersen, Blecha and Walker 1985) in agreement with the result of Schommer, 0lszewski and Aaronson (1984) for two intermediate-age clusters.

\subsection{Integrated Photometry and Spectra}

From early on, it was evident that some age discrimination could be made from UBV photometry alone (Gascoigne 1965). However a major procedural advance came from the work of Searle, Wilson and Bagnuolo (1980) who showed how it was possible to use intermediate band photometry to order the Magellanic Cloud clusters in a one-dimensional sequence. The character of the spectral variations along the sequence suggested that both chemical abundance and cluster age vary as the sequence is traversed so that clusters of the extreme type VII are the most metal poor and are old enough to come into the discussion in this paper. The method is not tied specifically to any particular photometric system. Frenk and Fall (1982) showed that UBV photometry suffices if 
variable interstellar reddening is not an important factor. The SWB classification system certainly seems to work. Clusters such as NGC 2210 and NGC 1786 classed as type VII were subsequently shown to have characteristics of old globular clusters (Graham 1981,1985; Hesser, McClure and Harris 1984). The SWB classification for Hodge 11 was confirmed when good color-magnitude diagrams became available.

Rabin (1982) has shown what can be accomplished from the direct analysis of integrated spectra themselves. From measurements of Balmer-line and $\mathrm{K}-\mathrm{l}$ ine strengths, he concluded that, at a given metallic line strength, the Cloud clusters have consistently stronger Balmer lines. There is a strong age dependence. Flower (1984) pointed out that the contribution of a small, stochastically variable number of red giant stars might distort this age dependence.

\subsection{RR Lyrae Variable Stars}

The presence of RR Lyrae stars in a cluster does not in itself guarantee advanced age. However, their appearance in many old clusters whose ages have been determined by other methods, underlines that they are, to say the least, useful flags which can easily be spotted with small telescopes (Graham and Nemec 1984). Nearly all SWB class VII clusters have RR Lyrae stars. A notable exception is Hodge 11 which has none simply because the horizontal branch is so blue that no stars are to be found in the instability strip. Walker (1984) has discussed the use of CCD observations of the RR Lyrae stars in NGC 2210 as indicators of the LMC distance.

\section{AGE SPREAD IN THE CLUSTER SYSTEM}

The age spread of the old globular clusters in the Magellanic Clouds seems to be greater than that in the Galaxy. This is not surprising in view of the existence of so many intermediate age populous clusters. It does mean that we have a good opportunity in the Magellanic Clouds to study the effects of age as well as of metallicity on the properties of a cluster a a whole. In the Galaxy the age differences are small and are very difficult to decouple from the abundance spread. In the Clouds this may not be the case. Both effects need to be understood if we are to make sense of the integrated properties of unresolved clusters in more distant systems.

It is appropriate to review here the characteristics of two clusters which have been studied in considerable detail in recent years. They are NGC 2257 in the Large Cloud and NGC 121 in the Small Cloud. Color-magnitude diagrams which extend below the main sequence turnoff have been published, surveys have been made for RR Lyrae stars and good abundance determinations are available for individual cluster members. Some properties are summarised in Table I. 
Table I

Comparison of Two Old Clusters

\begin{tabular}{|cccc|}
\hline & NGC 121 (SMC) & NGC 2257 (LMC) & Sources \\
\hline Age (Gyr) & $11 \pm 1$ & $15 \pm 2$ & 1,2 \\
{$[\mathrm{Fe} / \mathrm{H}]$} & $-1.4 \pm 0.1$ & $-2.0 \pm .2$ & 1,2 \\
$\mathrm{P}_{\mathrm{ab}}$ (RR Lyr) & $0.55 \mathrm{~d}$ & $0.58 \mathrm{~d}$ & 3,4 \\
$\mathrm{H}$. Branch & red & blue & 5,6 \\
Pec. Stars & $1 \mathrm{C}, 1$ SRd & $\begin{array}{c}\text { Double mode } \\
\text { RR Lyraes }\end{array}$ & 7,4 \\
\hline
\end{tabular}

Ref erences to Table I

1. Stryker, L.L., Da Costa, G.S. and Mould, J.R. 1985, Astrophys J. 298, p. 544 .

2. Stryker, L.L. 1983, Astrophys. J. 266, p. 82.

3. Graham, J.A. 1975, Pub. A.S.P., 87, p.641.

4. Nemec, J.M., Hesser, J.E. and Ugarte, P. 1985, Astrophys J. Suppl. 57, p. 287 .

5. Tifft, W.G. 1963, Monthly Notices Roy. Astron. Soc. 125, p.199.

6. Gascoigne, S.C.B. 1966, Monthly Notices Roy. Astron. Soc. 134, p. 59.

7. Feast, M.W. and Lloyd Evans, T. 1973, Monthly Notices Roy. Astron. Soc. 164 , p. 15P. 


\section{DYNAMICS OF THE OLD CLUSTER SYSTEM}

No review of the cluster system of the Magellanic Clouds would be complete without reference to the famous paper by Freeman, Illingworth and Oemler (1973) on the kinematics of the globular cluster system of the Large Magellanic Cloud. They were able to show that the youngest clusters formed a flattened system with low line-of-sight velocity dispersion and that this system shared the rotation solutions previously found for the young stars and the associated HI and HII. In addition they found that the older clusters with ages $>1$ Gyr also appeared to rotate as a flattened disk system but with a different line of nodes. This peculiarity persists to the oldest clusters discussed in this paper, but with less certainty owing to the small size of the sample. The question is raised as to whether, in view of this result, the Large Cloud has a halo population in the dynamical sense at all. Evidence to date is still fragmentary but it does suggest that the old stars may also be confined to a disk like structure (Graham 1975a, 1975b, 1977). We are conscious of the need to get velocity information for many more old objects but we should beware of trying to force-fit too much order and regularity into a galaxy which is certainly distorted and warped (Alvarez, Aparici and May 1987) especially in regions far from what little mass concentration there is in the central bar.

From dynamical arguments, Chun (1978) and Elson and Freeman (1985) suggest that the old clusters, NGC 1835, NGC 2257 and NGC 2210, may have masses $\left(\approx 5 \times 10^{4} \mathrm{M}_{\Theta}\right)$ which are low compared to those of globular clusters in our galaxy.

\section{SUMMARY REMARKS}

Looking towards the future both in this symposium and, in the longer term, towards the Hubble Space Telescope and beyond, I want to reiterate the importance of studying in detail the characteristics of globular clusters near at hand if we are to understand the integrated properties of distant globulars in remote galaxies. We need to know more precisely how abundance and age correlate with other properties such as the height of the horizontal branch above the main sequence turn-of $f$ and about the morphology of color magnitude diagrams as a whole. All these are important factors which determine the integrated characteristics. A good start has been made by Gascoigne, Bessell and Freeman (1981) and by Cohen (1982). It is not as simple a job as it looks. Gascoigne (quoted by Stryker 1983) has pointed out that a large fraction of the giants near a cluster can be field stars. In the Magellanic Clouds there are several cases where the cluster stars are distinctly older or younger than the surrounding field stars (Stryker 1983; Mould, Da Costa and Crawford 1984; Rich, Da Costa and Mould 1984) so considerable care has to be exercised in choosing the program stars and it is important to observe more than one or two stars per cluster to avoid the occasional interloper from the field. High weight data for well studied clusters are needed to make the foundation a solid one. 
ACKNOWLEDGEMENT

I would like to thank Johannes Andersen for bringing me up-to-date on some references that I had earlier missed.

\section{REFERENCES}

Alvarez, H., Aparici, J. and May, J. 1987 Astron. Astrophys., in press.

Andersen, J., Blecha, A. and Walker, M. F. 1984 Monthly Notices Roy. Astron. Soc., 211, 695 .

Andersen, J., Blecha, A. and Walker, M. F. 1985 Astron. Astrophys., $150, \mathrm{~L} 12$.

Arp, H. C. 1961 Science, 134, 810.

Chun, M. S. 1972 Astron. J., 82, 1062.

Cohen, J. G. 1982 Astrophs, J., 258, 143.

Elson, R. A. W. and Freeman, K. C. 1985 Astrophys. J., 288, 521.

Flower, P. J. 1984 in IAU Symposium No. 108, Structure and Evolution of the Magellanic Clouds, S. van den Bergh and K. S. de Boer, eds., Reidel, Dordrecht, p. 31.

Freeman, K. C., Illingworth, G. and Oemler, A. 1983 Astrophys. J., 272,488 .

Gascoigne, S. C. B. 1962 in Problems of Extra-Galactic Research, G. C. McVittie, ed., Macmillan, New York, p. 49.

Gascoigne, S. C. B. 1965 in Symposium on the Magellanic Clouds, J. V. Hindman and B. E. Westerlund, eds., Mount Stromlo Observatory, p. 66 .

Gascoigne, S. C. B. 1966 Monthly Notices Roy. Astron. Soc., 134,59 .

Gascoigne, S. C. B., BEsse11, M. S. and Norris, J. E. 1981 in IAU Colloquium No. 68, Astrophysical Parameters for Globular Clusters, A. G. D. Philip and D. S. Hayes, eds., L. Davis Press, Schenectady, p. 223.

Graham, J. A. 19875a Publ, Astron, Soc, Pacific, 87, 641.

Graham, J. A. 1975b Irish Astron. J., 12, 45.

Graham, J. A. 1977 Publ, Astron, Sco. Pacific, 89, 425.

Graham, J. A. and Nemec, J. M. 1984 in IAU Symposium No. 108. Structure and Evolution of the Magellanic Clouds, S. van den Bergh and $\mathrm{K}$. S. de Boer, eds., Reidel, Dordrecht, p. 37.

Hesser, J. E., McClure, R. D. and Harris, W. E. 1984 in IAU Symposium No, 108, Structure and Evolution of the Magellanic Clouds,

S. van den Bergh and K. S. de Boer, eds., Reide1, Dordrecht, p. 47.

Mould, J. and Aaronson, M. 1980 Astrophys. J., 240, 464.

Nemec, J. M. , Hazen-Liller, M. L. and Hesser, J. E. 1985 Astrophys. J. Supp1., 57, 329.

Nemec, J. M. Hesser, J. E. and Ugarte, P. 1985 Astrophys, J. Supp1, 57, 287.

Rabin, D. 1982 Astrophys. J,, 261, 85.

Rich, R. M., Da Costa, G. and Mould, J. 1984 Astrophys. J., $286,517$. 
Schommer, R. A., Olszewski, E. W. and Aaronson, M. A. 1984 Astrophys. J. (Letters), 285, L53.

Searle, L., Wilkenson, A. and Bagnuolo, W. G. 1980 Astrophys. J., $239,803$.

Shapley, H. 1930 in Star Clusters, McGraw-Hill, New York, p. 183.

Shapley, H. 1956 American Scientist, 44, 73.

Stryker, L. L. 1983 Astrophys. J., 266, 82. Stryker, L. L.,

Nemec, J. M. , Hesser, J. E. and McClure, R. D. 1984

in IAU Symposium No. 108, Structure and Evolution of the

Magellanic Clouds, S. van den Bergh and K. S. de Boer, eds., Reidel, Dordrecht, p. 43.

Suntzeff, N. B., Friel, E., Klemola, A., Kraft, R. P. and Graham, J. A. 1986 Astron. J., 91, 275.

Walker, A. R. 1985 Monthly Notices Roy. Astron. Soc., 212, 343.

Walker, M. F. 1979 Monthly Notices Roy. Astron. Soc., 186, 767. 


\section{DISCUSSION}

COHEN: Private communication from Freeman indicates that he no longer believes the model put forward by Freeman, Illingworth and Oemler where the older clusters form à disk inclined with respect to the younger clusters and the HI gas.

ZINN: Has Ken Freeman or anyone else evidence for a halo population in the LMC from kinematics?

COHEN: The existing radial velocities for the old LMC clusters are probably not accurate enough for a definite answer. I am planning to determine the velocities of a higher accuracy.

RICHER: A comment: We have observed the velocities of a number of hydrogen deficient carbon stars in the LMC which appear to be old when we compare them with those known in the Galaxy. Their dynamics can not be fit to that of a disk whether that shown by HI or any other. This is in disagreement with the Freeman, Oemler and Illingworth result.

SEGGEWISS; There is a recent paper by Elson and Freeman (1985) dealing with mass determination. They found a range of $2 \times 10^{4}-7 \times 10^{4} \mathrm{M}$. for old globular LMC clusters. These values are close to Galactic globular clusters with a mean total mass of $7 \times 10^{4} \mathrm{M}_{0}$ for the more star-rich and compact ones. 